\title{
O tamanho dos implantes atualmente disponíveis para a artroplastia total do quadril está adequado à nossa população?*
}

\section{Is the Size of the Currently Available Implants for Total Hip Arthroplasty Adequate for Our Population?}

\author{
Thiago Sampaio Busato ${ }^{1}$ Gabriella Maira Rodrigues Barbosa ${ }^{1}$ Anderson Emanuel Kumandala Velho ${ }^{1}$ \\ Gladyston Roberto Matioski Filho ${ }^{1}$ Lucas Dias Godoi ${ }^{1}$ Juan R.V. Capriotti ${ }^{1}$ \\ ${ }^{1}$ Serviço de Cirurgia do Quadril, Hospital Angelina Caron, Campina \\ Grande do Sul, Paraná, PR, Brasil \\ Rev Bras Ortop 2019;54:447-452. \\ Endereço para correspondência Thiago Sampaio Busato, MD, Serviço \\ de Cirurgia do Quadril, Hospital Angelina Caron, Rodovia do Caqui \\ 1.150, Campina Grande do Sul, Paraná PR, 83430-000, Brasil \\ (e-mail: tsbusato@hotmail.com).
}

\section{Resumo}

\section{Palavras-chave}

- artroplastia de quadril

- fraturas do quadril

- próteses de quadril

- desenho de prótese
Objetivo Definir se as dimensões de alguns implantes de fabricação nacional e estrangeira utilizados nas cirurgias de artroplastia do quadril estão adequadas ao perfil antropométrico da população brasileira.

Métodos Estudo retrospectivo de pacientes submetidos a cirurgia primária de substituição artroplástica total do quadril. Foram excluídos deste estudo pacientes com alterações morfológicas femorais ou acetabulares que pudessem influenciar na escolha do tamanho dos implantes, tais como sequelas de displasia, trauma, entre outras. Foram incluídos neste estudo dois implantes de fabricação nacional e seis modelos importados. Todos os pacientes foram operados pela mesma equipe, por um dos quatro autores seniores ou sob sua supervisão direta, seguindo a mesma técnica cirúrgica. Os dados foram estatisticamente analisados em relação a gênero, idade, tipo de fixação e modelo dos implantes.

Resultados A análise estatística de 682 quadris submetidos a artroplastia total demonstrou que 2 modelos de hastes femorais cimentadas e 1 de haste não cimentada não estão perfeitamente adequados à morfologia femoral da população estudada, pois, na maioria dos casos, nestes 3 implantes, foi utilizado o menor tamanho disponível, resultando em uma curva não gaussiana. O diâmetro médio do acetábulo nativo foi de $54 \mathrm{~mm}$ nos homens e de $52 \mathrm{~mm}$ nas mulheres.

Conclusão Dos oito modelos estudados, cinco se mostraram adequados à população estudada. Outros três modelos disponíveis em nosso mercado (dois nacionais e um importado) parecem necessitar de um escalonamento mais adequado. Destacamos que são necessários estudos antropométricos do quadril da população brasileira para dar subsídios científicos ao desenho ideal dos implantes para o nosso mercado.

Trabalho desenvolvido no Serviço de Cirurgia do Quadril, Hospital Angelina Caron, Campina Grande do Sul, Paraná, PR, Brasil.

(D) Thiago Sampaio Busato's ORCID is https://orcid.org/0000-00027165-5173.

recebido

21 de Setembro de 2018

aceito

2 de Fevereiro de 2019
DOI https://doi.org/

10.1055/s-0039-1694713. ISSN 0102-3616.
Copyright $\odot 2019$ by Sociedade Brasileira License terms de Ortopedia e Traumatologia. Published by Thieme Revnter Publicações Ltda, Rio de Janeiro, Brazil 


\section{Abstract}

\section{Keywords}

- arthroplasty, replacement, hip

- hip fractures

- hip prosthesis

- prosthesis design
Objective To determine if the dimensions of selected national and imported implants used in total hip arthroplasty surgeries are adequate regarding the anthropometric profile of the Brazilian population.

Methods A retrospective study of patients submitted to primary total hip arthroplasty. Patients with femoral or acetabular morphological deformities that could influence the choice of implant size, such as dysplasia, previous fractures, among others, were excluded from the study. Two national implants and six imported ones were included. Every patient was treated by the same group, by one of the four senior authors or under their strict supervision, following the same surgical technique. The data were analyzed regarding gender, age, fixation type and implant model.

Results The analysis of 682 hips submitted to total hip arthroplasty demonstrated that 2 models of cemented femoral stems and 1 of non-cemented stem did not seem to be perfectly adequate for the femoral morphology of the studied population, since, in the majority of cases, these 3 implants were used in the smallest size available, resulting in a non-Gaussian curve. The mean diameter of the native acetabulum was 54 $\mathrm{mm}$ in men and $52 \mathrm{~mm}$ in women.

Conclusion Out of the eight models studied, five were shown to be adequate for the studied population. The other three models available in our market (two national models and one imported model) seemed to need more appropriate sizing. We emphasize that anthropometric studies of the Brazilian hip are necessary to give scientific subsidies to the ideal design of the implants for our market.

\section{Introdução}

A artroplastia total do quadril representa um dos procedimentos cirúrgicos mais revolucionários e bem-sucedidos da medicina moderna, proporcionando aos pacientes alívio dos sintomas e melhora na qualidade de vida. ${ }^{1}$

O conceito básico do procedimento consiste na substituição da superfície articular degenerada por componentes protéticos, que apresentam inúmeras variações de design, tipos de fixação e de pares tribológicos. Os tipos de substituições artroplásticas geralmente dividem-se em três grupos distintos, de acordo com o tipo de fixação dos implantes: cimentados, não cimentados, e híbridos (nos quais apenas um dos componentes é fixado usando cimento ósseo).

É de suma importância a escolha adequada dos implantes a serem utilizados na cirurgia, sendo respeitado o planejamento pré-operatório individualizado para cada paciente, com o objetivo de restaurar perfeitamente a biomecânica e a função do quadril. 0 presente estudo surgiu de uma percepção prática de que, em algumas situações, alguns modelos de implantes pareciam ser muito grandes para nosso paciente, sendo por vezes necessária a adaptação do paciente ao implante e não o contrário, que seria o desejável.

Após uma revisão da literatura, os autores não encontraram dados que descrevessem o padrão de tamanho médio dos implantes utilizados nas cirurgias de artroplastia do quadril na população brasileira, nem estudos antropométricos específicos sobre o quadril da mesma população. A importância de se obter estes dados abarca desde a possibilidade de melhor adequação dos implantes disponíveis, até o impacto financeiro, envolvendo volumes de estoque e assim por diante.

\section{Materiais e Métodos}

Após a aprovação pelo Comitê de Ética em Pesquisa de nossa instituição, foram avaliados retrospectivamente para este estudo prontuários e registros médicos de pacientes submetidos a procedimento de substituição primária artroplástica total de quadril, operados por um dos quatro cirurgiões autores seniores deste estudo ou sob sua supervisão direta, no período compreendido entre janeiro de 2014 e janeiro de 2018. Foram revisadas descrições cirúrgicas e registros de órteses, próteses e materiais especiais (OPME), e levantados todos os tamanhos de implantes utilizados nessas cirurgias.

O seguinte critério de inclusão foi utilizado na seleção da amostra: pacientes submetidos a artroplastia primária total do quadril, sem deformidades prévias.

Os critérios de exclusão foram fatores secundários que poderiam influenciar na seleção do tamanho do implante, tais como: sequela de displasia do desenvolvimento do quadril, sequela de fratura do acetábulo, sequela de fratura do fêmur proximal (excluindo-se colo e cabeça femoral), artroplastias de revisão, osteotomias pélvicas ou femorais, e/ ou cirurgia de conversão ou desartrodese.

Todos os pacientes incluídos neste estudo foram submetidos a avaliação pré-operatória de rotina (exames laboratoriais, radiografias, protocolo pré-operatório da instituição, planejamento pré-operatório por meio de imagens radiográficas, e uso de templates analógicos). O planejamento pré-operatório, rotineiro em nosso serviço, permite uma antecipação em termos de largura da haste, offset, e diâmetro acetabular. Os pacientes foram posicionados em decúbito lateral com auxílio de estabilizadores pélvicos; foi realizada abordagem ao quadril 
pelo acesso póstero-lateral, e usadas referências anatômicas intraoperatórias para o posicionamento adequado dos componentes. Foram utilizados implantes cirúrgicos de fabricação nacional e importada. Os pacientes foram avaliados de acordo com o gênero, idade, tipo de fixação e modelo dos componentes utilizados.

É importante notar que, para este estudo, considerou-se o diâmetro da última fresa acetabular utilizada durante o preparo do leito ósseo para fins de cálculo do diâmetro acetabular médio.

Neste estudo, no qual incluímos oito modelos de haste femoral, a escolha do tipo e marca de implante deu-se por disponibilidade, preferência pessoal do cirurgião, e pelo índice de Dorr. Como cada fabricante tem uma nomenclatura diferente para o tamanho de suas hastes femorais, criou-se uma tabela de correlação, de modo que convencionamos que o menor implante disponível de cada sistema seria chamado pela letra "A", o seguinte, pela letra "B", e assim por diante, de acordo com os tamanhos disponíveis segundo os prospectos de cada fabricantes (-Anexo 1).

Os diferentes modelos e tamanhos de implantes femorais utilizados foram analisados percentualmente de acordo com o sistema de correlação alfabética desenvolvido. Suas curvas de distribuição foram traçadas para que se permitisse a análise de sua semelhança a uma curva normal ou Gaussiana.

\section{Resultados}

No período abrangido pelo estudo, foram identificadas 842 artroplastias primárias, e foram selecionados 682 procedimentos que atendiam aos critérios de inclusão, $326 \mathrm{em}$ pacientes do gênero masculino (48\%) e 356 em pacientes do gênero feminino (52\%) da amostra. A idade média da amostra foi de 53 anos, variando entre 17 a 89 anos.

O tamanho do acetábulo no sexo masculino variou entre $46 \mathrm{~mm}$ e $62 \mathrm{~mm}$, tendo uma média de $54 \mathrm{~mm}$. Em relação ao sexo feminino variou entre $44 \mathrm{~mm}$ e $58 \mathrm{~mm}$, com média de $52 \mathrm{~mm}$.

Foram implantados 482 (70,7\%) hastes femorais cimentadas e 200 (29,3\%) hastes não cimentadas. Nas substituições femorais, foram utilizados os sistemas: CStem e Summit (DePuy Synthes, Raynham, MA, EUA); Exeter e Accolade (Stryker, Portage, MI, EUA); Trilliance e Bicontact (Aesculap, Center Valley, PA, EUA ); e Alfa e Co-10 (Baumer). As hastes cimentadas utilizadas foram todas do tipo polida e com geometria em cunha, sendo três em dupla cunha (Exeter, Trilliance e Alpha) e uma em tripla cunha (Cstem). As hastes não cimentadas utilizadas foram todas de fixação metafisária, sendo três do tipo fit and fill (Summit, Bicontact e Co-10) e uma do tipo taper (Accolade). Foram implantadas 258 hastes importadas, sendo 95 em pacientes do sexo masculino e 163 do sexo feminino.

A - Tabela 1 mostra a descrição da amostra populacional encontrada em cada sistema.

Comparando-se o tamanho das hastes cimentadas importadas com a haste nacional, observamos uma distribuição mais homogênea em relação aos tamanhos usados nos materiais importados, conforme indica a -Figura 1. Houve predomínio de uso dos menores tamanhos disponíveis no material nacional.

Comparando-se o tamanho das hastes não cimentadas importadas com a haste nacional, também observamos uma distribuição mais homogênea em relação aos tamanhos usados no material importado, conforme mostra a - Figura 2. Também houve predomínio dos menores tamanhos disponíveis no material nacional.

Para que a distribuição dos tamanhos das hastes usadas seja considerada adequada a uma determinada população, deve ser encontrado um padrão de curva de distribuição normal ou gaussiana. Nesta distribuição, a maioria da população estaria no meio da curva, ou seja, com os implantes de tamanho

Anexo 1 Padronização da nomenclatura

\begin{tabular}{|l|l|l|l|l|l|l|l|l|}
\hline & \multicolumn{4}{l}{ HASTES FEMORAIS CIMENTADAS } & \multicolumn{4}{l|}{ HASTES FEMORAIS NÃO CIMENTADAS } \\
\hline & CSTEM & EXETER & TRILLIANCE & ALFA & SUMMIT & ACCOLADE & BICONTACT & CO-10 \\
\hline A & CDH & 30 & 8 & 0 & 1 & 0 & 9 & 9 \\
\hline B & 1 & 33 & 10 & 0,5 & 2 & 1 & 10 & 11 \\
\hline C & 2 & 35,5 & 12 & 1 & 3 & 2 & 11 & 13 \\
\hline D & 3 & 0 & 14 & 2 & 4 & 3 & 12 & 15 \\
\hline E & 4 & 1 & 16 & 3 & 5 & 4 & 13 & 17 \\
\hline F & 5 & 2 & N/D & N/D & 6 & 5 & 14 & 19 \\
\hline G & 6 & 3 & N/D & N/D & 7 & 6 & 15 & N/D \\
\hline H & 7 & 4 & N/D & N/D & 8 & 7 & 16 & N/D \\
\hline I & 8 & 5 & N/D & N/D & 9 & 8 & 17 & N/D \\
\hline J & N/D & 6 & N/D & N/D & 10 & 9 & 18 & N/D \\
\hline K & N/D & N/D & N/D & N/D & N/D & 10 & 19 & N/D \\
\hline L & N/D & N/D & N/D & N/D & N/D & 11 & 21 & N/D \\
\hline
\end{tabular}

Nota: Sistema alfabético para correlação das diferentes nomenclaturas dos tamanhos disponíveis.

Abreviaturas: $\mathrm{CDH}$, Congenital Dislocation of the Hip; N/D, tamanho não disponível. 
Tabela 1 Amostra populacional

\begin{tabular}{|l|l|l|l|l|l|l|l|l|}
\hline & \multicolumn{4}{|l|}{ HASTES CIMENTADAS } & \multicolumn{4}{l|}{ HASTES NÃO CIMENTADAS } \\
\hline & CSTEM & EXETER & TRILLIANCE & ALFA & SUMMIT & ACCOLADE & BICONTACT & CO-10 \\
\hline N TOTAL & 55 & 73 & 130 & 224 & 44 & 19 & 69 & 68 \\
\hline $\begin{array}{l}\text { N MASCULINO } \\
(\%)\end{array}$ & $21(23,3)$ & $17(32,8)$ & $57(43,8)$ & $99(44,1)$ & $40(90,9)$ & $18(94,7)$ & $30(43,4)$ & $61(89,7)$ \\
\hline $\begin{array}{l}\text { N FEMININO } \\
(\%)\end{array}$ & $34(76,7)$ & $56(61,8)$ & $73(56,1)$ & $125(55,8)$ & $4(9,1)$ & $1(5,3)$ & $39(56,5)$ & $7(10,2)$ \\
\hline $\begin{array}{l}\text { IDADE MÉDIA } \\
\text { (MÍN-MÁX) }\end{array}$ & $66(22-89)$ & $62(42-89)$ & $56(27-82)$ & $64(30-87)$ & $49(23-73)$ & $48(32-59)$ & $52(17-87)$ & $64(30-87)$ \\
\hline
\end{tabular}

HASTE CIMENTADA NACIONAL

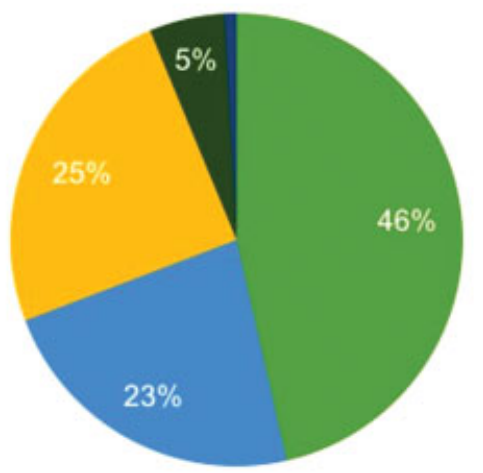

$A \odot B \bigcirc C \bigcirc D \bigcirc E$

\section{HASTES CIMENTADAS IMPORTADAS}

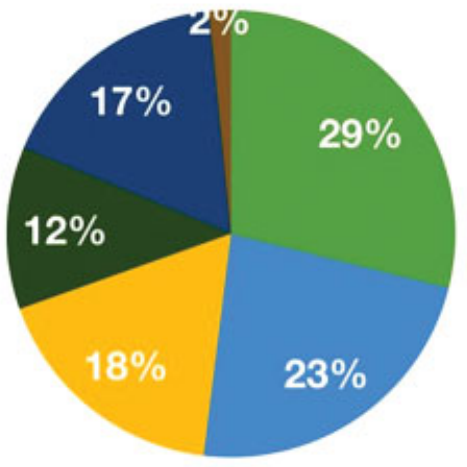

A

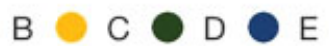

$E \cap F \bigcirc G$

Fig. 1 Distribuição percentual dos tamanhos utilizados da haste cimentada nacional e das hastes cimentadas importadas.

HASTE NÃO CIMENTADA NACIONAL

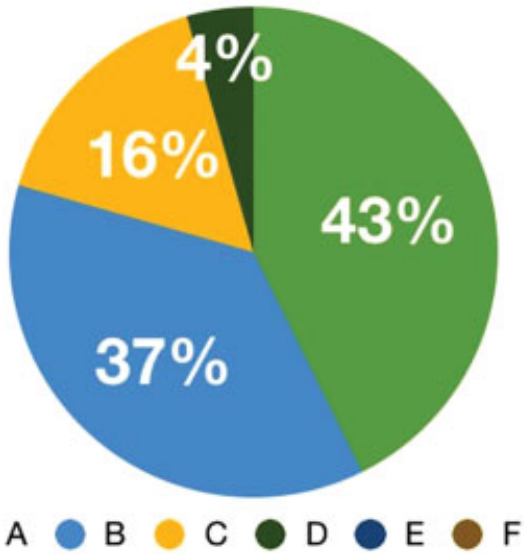

\section{HASTES NÃO CIMENTADAS IMPORTADAS}

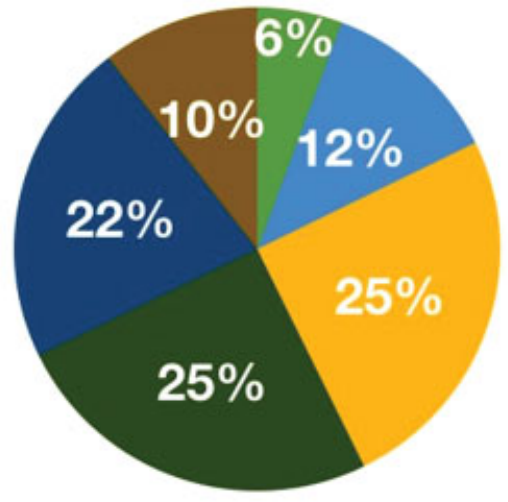

A

$B$

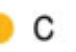

D

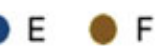

Fig. 2 Distribuição percentual dos tamanhos utilizados da haste não cimentada nacional e das hastes não cimentadas importadas.

médio, e uma pequena parcela estaria nas extremidades, ou seja, com implantes de tamanho pequeno ou grande.

As porcentagens de cada tamanho utilizado de implante cimentado foram tabeladas e convertidas em curvas, conforme indica a - Figura 3. Nota-se que os implantes Trilliance e Alfa não demonstraram um padrão de curvas de distribuição normal ou gaussiano. Este fato possivelmente denota que, nestes dois implantes, os tamanhos disponíveis encontram- se superdimensionados para a população estudada, pois foram usados os menores tamanhos disponíveis na maioria dos casos. As hastes Exeter e Cstem exibiram uma distribuição mais próxima de curvas Gaussianas, o que representaria um dimensionamento mais adequado dos implantes a esta população.

As porcentagens de cada tamanho utilizado de implante não cimentado também foram tabeladas e convertidas em curvas, conforme mostra a - Figura 4. Nota-se que apenas o 


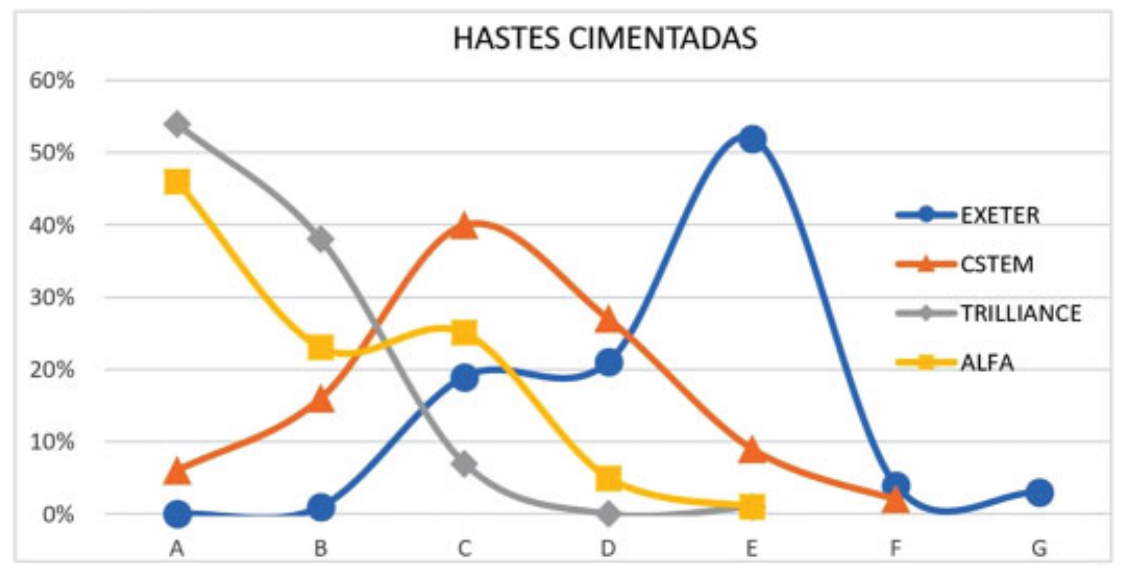

Fig. 3 Distribuição de tamanhos utilizados da haste cimentada nacional e das hastes cimentadas importadas.

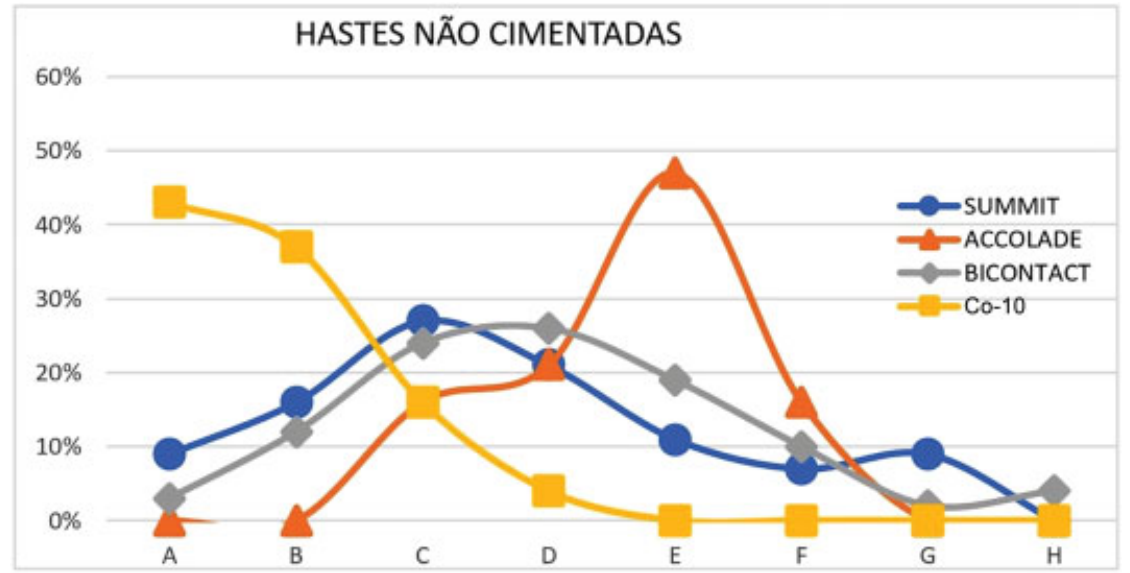

Fig. 4 Distribuição de tamanhos utilizados da haste não cimentada nacional e das hastes não cimentadas importadas.

implante Co-10 não demonstrou um padrão de curva de distribuição normal ou gaussiano. Isto possivelmente demonstra que, neste implante, os tamanhos disponíveis encontram-se superdimensionados para a população estudada. As hastes Summit, Accolade e Bicontact exibiram um comportamento mais próximo de curvas gaussianas, o que representaria um dimensionamento mais adequado dos implantes.

\section{Discussão}

A artroplastia de substituição do quadril tem como uns dos seus objetivos primordiais restaurar a anatomia e função da articulação. ${ }^{1}$ Com base nisso, é de suma importância o conhecimento da influência do gênero, da idade e das patologias articulares de base no padrão anatômico da articulação. ${ }^{2,3}$ Estudos anatômicos têm demonstrado diferenças significativas entre os gêneros, principalmente na anatomia femoral. ${ }^{4-8}$ Mulheres tendem a apresentar ângulo cervicodiafisário menor, offset menor, colo femoral mais curto, diáfise mais fina, e maior anteversão femoral. ${ }^{9}$ Wang et $\mathrm{al}^{6}{ }^{6}$ em estudo de tomografia da pelve, encontraram diferenças significativas entre os gêneros no que diz respeito à profundidade da articulação, versão acetabular e diâmetro acetabular. Segundo seus resultados, no gênero feminino o acetábulo tende a ser mais profundo e antevertido, porém, de menor diâmetro quando comparado ao do gênero oposto.

Estudo antropométrico de Noble et $\mathrm{al}^{5}$ demonstrou que a maioria dos parâmetros anatômicos femorais se aproximam da distribuição gaussiana. Desta forma, seria justo esperar que o tamanho dos implantes femorais também devesse se distribuir desta maneira, para uma melhor adequação à anatomia. $O$ fato de que as curvas de distribuição de alguns dos implantes de nosso estudo não resultaram em um padrão comparável a uma curva de distribuição gaussiana vai de encontro a estes achados, inferindo que é necessário um dimensionamento mais adequado destes implantes à nossa população.

Sendo o Brasil um país miscigenado, ${ }^{10}$ seria desejável adequar os tamanhos e modelos dos implantes às características demográficas da população nacional. Infelizmente, estes tipos de estudos ainda não estão disponíveis com relação à nossa população.

0 presente estudo tem algumas limitações inerentes ao desenho retrospectivo, bem como ao viés de seleção do tipo de implante com base em sua disponibilidade e preferência do cirurgião. Além disso, engloba realidades distintas de pacientes atendidos pelo Sistema Único de Saúde e por meio de convênios médicos. Nota-se, portanto, que por estes 
motivos houve certa discrepância entre as subamostras em relação a gênero e tipo de implante. Não foi realizada análise crítica inter ou intraobservador da qualidade da implantação ou do dimensionamento dos implantes.

Por outro lado, os pontos positivos deste estudo são o número representativo e diversificado de indivíduos e de implantes na amostra, o que reflete a realidade de um serviço de alto fluxo, referência em cirurgia do quadril, e que presta atendimento aos setores público e privado. Além disso, a hipótese inicial foi corroborada pelos achados, haja vista o desenho não gaussiano de algumas curvas. Os dados deste estudo podem nortear os fabricantes e alertar os colegas cirurgiões sobre implantes que exigem maior cuidado, bem como incentivar a pesquisa e o desenvolvimento de estudos antropométricos do quadril do brasileiro, possibilitando uma melhor adequação dos implantes disponíveis.

Quanto ao tamanho dos implantes femorais, há uma vasta gama de materiais disponíveis no mercado brasileiro e internacional, e seu dimensionamento e numeração variam de acordo com o fabricante. Porém, nota-se que, apesar de alguma variação na numeração do tamanho dos implantes, existe uma tendência ao uso de implantes de menor tamanho em $\sim 70$ a $80 \%$ dos procedimentos cirúrgicos, independente do tipo de fixação, quando se trata de implantes de fabricação nacional. O contrário foi observado nos dados das hastes importadas com melhor adequação anatômica e utilização mais ampla e variada das numerações de hastes disponíveis nas mais diversas marcas, com exceção de um implante apenas.

Parece claro, após a análise dos achados desta investigação, que são necessários estudos antropométricos populacionais brasileiros para melhor adequação de alguns implantes nacionais e importados disponíveis em nosso meio para a artroplastia do quadril.

\section{Conclusão}

Três dos oito implantes estudados em nossa amostra não estão adequadamente escalonados para a população estudada.

Conflito de Interesses

Os autores declaram não haver conflitos de interesses.

\section{Referências}

1 Learmonth ID, Young C, Rorabeck C. The operation of the century: total hip replacement. Lancet 2007;370(9597):1508-1519

2 Bourne RB, Rorabeck CH. Soft tissue balancing: the hip. J Arthroplasty 2002;17(04, Suppl 1):17-22

3 Charles MN, Bourne RB, Davey JR, Greenwald AS, Morrey BF, Rorabeck $\mathrm{CH}$. Soft-tissue balancing of the hip: the role of femoral offset restoration. Instr Course Lect 2005;54:131-141

4 Nieves JW, Formica C, Ruffing J, et al. Males have larger skeletal size and bone mass than females, despite comparable body size. J Bone Miner Res 2005;20(03):529-535

5 Noble PC, Alexander JW, Lindahl LJ, Yew DT, Granberry WM, Tullos HS. The anatomic basis of femoral component design. Clin Orthop Relat Res 1988;(235):148-165

6 Wang SC, Brede C, Lange D, et al. Gender differences in hip anatomy: possible implications for injury tolerance in frontal collisions. Annu Proc Assoc Adv Automot Med 2004;48:287-301

7 Kaptoge S, Dalzell N, Loveridge N, Beck TJ, Khaw KT, Reeve J. Effects of gender, anthropometric variables, and aging on the evolution of hip strength in men and women aged over 65 . Bone 2003;32(05):561-570

8 Sariali E, Mouttet A, Pasquier G, Durante E. Three-dimensional hip anatomy in osteoarthritis. Analysis of the femoral offset. J Arthroplasty 2009;24(06):990-997

9 Traina F, De Clerico M, Biondi F, Pilla F, Tassinari E, Toni A. Sex differences in hip morphology: is stem modularity effective for total hip replacement? J Bone Joint Surg Am 2009;91(Suppl 6):121-128

10 IBGE - Instituto Brasileiro de Geografia e Estatística. Indicadores Sociodemográficos e de Saúde no Brasil-2009. Disponível em: http://biblioteca.ibge.gov.br/visualizacao/livros/ liv42597.pdf 